\title{
Efectividad de un programa de estimulación del lenguaje sobre la capacidad de solución de problemas de niños de seis años escolarizados
}

\author{
Effectiveness of a Language Stimulation Program on the Ability \\ of Solving Problems in School Children Six Years
}

\author{
Eficácia de um programa de estimulação da linguagem na capacidade \\ de resolução de problemas de crianças de escolas seis anos
}

\author{
Juan Pablo Sánchez Escudero, Johny Villada Zapata. ${ }^{a}$ \\ a Departamento de Psicología, Universidad de Antioquia, Colombia. \\ Fono: 094-2195763. Correos electrónicos: jupanet12@msn.com, johnyvillada@gmail.com
}

\begin{abstract}
RESUMEN
El presente estudio buscó establecer la efectividad de un programa de estimulación del lenguaje sobre la capacidad de resolución de problemas en niños de seis años escolarizados. La muestra estuvo conformada por 22 niños (12 niñas y 10 niños) de un colegio del oriente antioqueño (Colombia). El diseño utilizado fue cuasi-experimental ABA de casos y controles con evaluación Pre-test y Pos-test. El grupo de "casos" fue expuesto a 10 sesiones de estimulación del lenguaje durante un periodo de cinco semanas. El análisis intragrupal muestra que aunque no hay diferencias respecto al razonamiento usado (movimientos válidos y erróneos), los niños casos logran solucionar la situación problema en menos tiempo que los niños controles después de pasar por las sesiones de estimulación del lenguaje. El análisis intergrupal no muestra diferencias significativas, probablemente debido al poco tiempo de exposición de los niños al programa.
\end{abstract}

Palabras clave: solución de problemas, lenguaje, estimulación.

\begin{abstract}
This study aimed to establish the effectiveness of a language stimulation program on the problem solving ability in children of six years at school. The sample consisted of 22 children (12 girls and 10 boys) from a college in eastern Antioquia (Colombia). The design was quasi-experimental ABA case control with Pre-test and post-test. The group of "cases" was exposed to 10 sessions of language stimulation for a period of five weeks. Intragroup analysis shows that although there is no difference with the reasoning used (valid and erroneous movements), children-case study solve the problem situation in less time than the control children after going through the language stimulation sessions. The intergroup analysis shows no significant differences, probably due to the short time of exposure of children to the program.

Key words: problem solving, language, stimulation.
\end{abstract}

\section{RESUMO}

Objetivou-se estabelecer a eficácia de um programa de estimulação da habilidade da linguagem sobre a capacidade de crianças de seis anos e escolarizadas para solucionar problemas. A amostra foi composta por 22 crianças (12 meninas e 10 meninos), de uma faculdade no leste de Antioquia (Colômbia). O projeto era quase-experimental, de casocontrole ABA, com avaliação de pré-teste e pós-teste. O grupo de "casos" foi exposto a 10 sessões de estimulação da linguagem por um período de cinco semanas. Análise intragrupo mostra que, embora não haja diferenças entre o raciocínio utilizado (movimentos válidos e errados), pelas crianças que resolvem situações-problema em menos tempo do que aquelas que passaram por sessões de estimulação de linguagem. Tal análise não apresenta diferenças significativas entre os grupos por causa do curto tempo de exposição das crianças ao programa, provavelmente.

Palavras chave: solução de problemas, linguagem, estimulação. 


\section{INTRODUCCIÓN}

El aprendizaje basado en solución de problemas (SP), el procesamiento activo de la información y la estimulación de habilidades cognitivas, han logrado posicionarse como un tema central de la investigación, tanto para la pedagogía como para la psicología educativa (Rohde \& Thompson, 2007). Es por ello que se vienen desarrollando, en distintas partes del mundo, programas de estimulación que faciliten la SP, para que ésta se convierta en una herramienta que transversalice los procesos de enseñanza y apropiación del conocimiento (Dewey \& Bento, 2009).

Para Carretero y Asencio (2004) una SP ocurre cuando la actividad de un sujeto tiene un fin, pero no encuentra un camino preciso o bien definido hasta él. Para algunos autores como James (Cit. en Rains, 2003: 4), la habilidad para seguir metas a pesar de los obstáculos es una característica de la inteligencia.

En el estudio de la capacidad de SP en los seres humanos diversos son los enfoques, métodos y experimentos usados. La presente investigación retoma dos modelos que, aunque geográfica e históricamente distintos, podrían ser recuperados en conjunto para lograr perspectivas teóricas más amplias. Los modelos son el de procesamiento de la información propuesto por Simon, y el enfoque sociocultural de Vigotsky y Luria.

La primera perspectiva ha progresado a grandes pasos en lo referente a proporcionar explicaciones de los procesos utilizados en la SP relativamente bien estructurados (Simon, Cit. en Carretero, García y García, 1995: 203). La teoría describe al sujeto como un procesador de la información que interactúa con un ambiente de la tarea, entendida como la tarea tal como es explicada por el experimentador. El sujeto que soluciona el problema representa la situación en términos de un espacio del problema que es la manera subjetiva de considerar el ambiente de la tarea.

El autor propone cuatro leyes de estructura cualitativa, entendidas como proposiciones acerca de la estructura fundamental de un conjunto de fenómenos (Newell y Simon, Cit. en Carretero, García y García, 1995: 208), en este caso de la solución de problemas. Estas proposiciones son:

1. El Sistema de Procesamiento de la Información (SPI) humana es un sistema adaptativo, capaz de amoldar su conducta, dentro de límites amplios, a las exigencias de la tarea capaz de modificar su conducta a lo largo del tiempo a través del aprendizaje.

2. Las características del SPI son suficientes para determinar que el ambiente de la tarea se representará como un espacio del problema y que la solución del problema se realizará en éste.

3. La estructura del ambiente de la tarea determina las posibles estructuras del espacio del problema.

4. La estructura del espacio del problema determina los programas posibles que pueden utilizarse para la solución de problemas.

De acuerdo a esto, a partir del conocimiento del ambiente de la tarea, se pueden hacer predicciones, pero sólo incompletas, sobre las características del espacio del problema; y a partir de un conocimiento de éste, predicciones incompletas sobre la estrategia de la SP (Simon, Cit. en Carretero, García y García, 1995: 212).

El SPI opera casi por completo de manera serial, llevando a cabo un proceso a la vez, evidenciándose en la estrechez del foco atencional, las entradas y salidas de estos procesos 
se mantienen en una memoria a corto plazo. Esta descripción proviene de experimentos realizados por psicólogos durante las últimas décadas (Norman, Cit. en Carretero, García y García, 1995: 215). Los sujetos que resuelven problemas no muestran ninguna conducta que exija una búsqueda simultánea de partes inconexas del espacio del problema, por el contrario, la conducta requiere la forma de una búsqueda secuencial.

Para poder solucionar un problema, el sujeto debe hacerse una representación del ambiente de la tarea, dicha representación constituye el espacio del problema del sujeto. La estructura del espacio del problema direcciona la conducta de varias formas. Define los movimientos lícitos, define también la meta y de forma implícita el movimiento "lejos" o "cerca" de la meta, por último, interactúa con los límites de la memoria a corto plazo haciendo que algunos caminos de la solución resulten más fáciles de encontrar que otros (Simon, Cit. en Carretero, García y García, 1995: 218).

El espacio del problema más sencillo de una tarea, consiste en un conjunto de nodos generados por todos los movimientos válidos; cada uno de estos nodos debe ser considerado como un posible estado de conocimiento que el sujeto puede alcanzar. La búsqueda de la solución es un recorrido de un estado de conocimiento a otro, hasta que el nodo actual incluya la solución del problema.

Con esto se tiene que, dependiendo de las exigencias realizadas al sujeto, los espacios del problema pueden variar considerablemente de tamaño; "la relativa facilidad de la solución de un problema dependerá del éxito que haya tenido el sujeto a la hora de representarse en su espacio del problema las características críticas del ambiente de la tarea" (Simon, Cit. en Carretero, García y García, 1995: 202).

Los espacios del problema difieren tanto de tamaño como de estructura, dicha estructura permite hacer predicciones acerca de los estadios aún no conocidos del problema, evidencia de esto es la conciencia de los sujetos de "acercarse" a la meta cuando resuelven un problema. Cuando un sujeto llega a un nodo determinado, puede escoger un operador específico para llegar a otro nodo o estado de conocimiento, incluso puede desistir de ese nodo y usar otro operador para llegar a otro o volver sobre el anterior. El sujeto generalmente evalúa el nodo actual con base en la diferencia entre éste y la situación-meta, alcanzar un nodo que se diferencia menos de la situación deseada, lo que significa progreso, y escoger un operador que reduzca una diferencia determinada entre el nodo actual y la meta, constituyendo una técnica para resolver problemas.

Pasando al segundo modelo, el de la psicología histórico-cultural de Vygotsky, Luria, Leóntiev y seguidores, la solución de problemas aparece como una muestra perfecta de una función mental superior o proceso mental complejo (Montealegre, 2007). Desde las concepciones funcionalistas de Luria (Cit. en Molina, 2009: 2), el lenguaje juega un papel fundamental en la regulación de las funciones cognitivas y del pensamiento, de modo que la capacidad de solución de problemas (SP) no es ajena a este postulado.

Vygotsky (Cit. en Montealegre, 2007: 21) enfatiza en el lenguaje como un instrumento de planificación y regulación intelectual de la acción, y concluye que los infantes resuelven tareas prácticas con la ayuda de éste. En este planteamiento el autor tiene en cuenta varios factores: el primero es que la acción y el lenguaje son una misma función psicológica dirigida a la solución del problema planteado; el segundo es que para el niño, entre más compleja sea la tarea exigida y menos directa su solución, mayor es la importancia del lenguaje, llegando al punto de que si se le prohíbe al infante hablar, no podrá resolver el problema. 
Es así como en los postulados de Vygostky (Cit. en Ardila y Ostrosky-Solís, 2008: 4), un factor primordial en la organización sistemática de los procesos cognitivos superiores es la inclusión de elementos externos (objetos, signos, símbolos) propios de cada cultura, siendo uno de los primordiales el lenguaje. De modo que el papel de los instrumentos externos es el de establecer conexiones funcionales entre varios sistemas universales, logrando así una organización extracortical de las funciones mentales superiores, entendida como la interacción de factores biológicos y culturales en el desarrollo de la cognición humana.

El lenguaje, como elemento externo y cultural, cumple dicha función organizativa y permite la interacción de diversos sistemas corticales en pro del surgimiento de los procesos mentales superiores, entre los cuales se encuentra incluida la SP, lo cual permitiría inferir que ambos procesos se encuentran interrelacionados por lo menos en algún nivel. Ya Vygotsky lo planteaba en su obra Pensamiento y lenguaje, al decir que "Pensamiento y palabra se encuentran conectados por un vínculo primario. La conexión se origina, cambia y crece en el curso de la evolución" (1995: 159).

Solucionar problemas es una tarea tan comúnmente realizada que en ocasiones se obvia la cantidad de procesos implícitos a la hora de ejecutarla. Dicha capacidad se encuentra ya presente desde la primera infancia, sin embargo, tal como lo muestran los estudios de Piaget (1991), sufre cambios en el proceso de desarrollo del niño. Dicho de otra manera: "la relación entre pensamiento y palabra no es un hecho, sino un proceso, un continuo ir y venir del pensamiento a la palabra y de la palabra al pensamiento, y en él la relación entre pensamiento y palabra sufre cambios que pueden ser considerados como desarrollo en el sentido funcional" (Vygostky, 1995: 165).

Han sido varios los autores que se han interesado por estas relaciones entre lenguaje y solución de problemas. Kintsch \& Greeno (1985) proponen que para la comprensión de problemas aritméticos, el lenguaje se convierte en un elemento central en la construcción de representaciones cognitivas que facilitan la respuesta dirigida a la salida esperada. Setti \& Caramelli (2007) encontraron que el uso de la dimensión pragmática del lenguaje facilita la solución de problemas en los que se han dado datos irrelevantes. Rich, Miller \& DeTora, (2011) afirman que el uso del lenguaje hablado y escrito favorece la solución de problemas en el área de ciencias naturales, facilitando la metacognición. Fahim \& Pezeshki (2012) sostienen que las pruebas de lenguaje que se usan en la educación primaria, pueden ser modificadas para promover solución de problemas específicos y pensamiento crítico.

Gracias a este interés en las relaciones entre ambos procesos, se puede plantear la utilidad de usar el lenguaje como instrumento en la estimulación de las estrategias de solución de problemas en niños pequeños, lo cual constituye el objetivo de la presente investigación. Se tomaron específicamente niños de 6 años porque están empezando la educación formal y, al estar homogeneizados por edad, se tiene mayor control sobre esa variable que podría influir en los resultados de solución de problemas.

Ahora bien, siendo tan amplios ambos conceptos (SP y lenguaje) y tan compleja su conceptualización teórica, se hizo necesario establecer el foco de la estimulación. Siendo la SP un proceso lógico guiado por pasos, en los cuales es necesario integrar actividades a través del tiempo para encontrar la forma más adecuada de proceder, y teniendo en cuenta que la representación del tiempo en el lenguaje deriva de las representaciones espaciales evidenciándose en palabras como "antes", "ahora" y "después" cuyo uso requiere del manejo adecuado del lenguaje (Ardila y Ostrosky-Solís, 2008), se realizó hincapié entonces en actividades de lenguaje que incluían la secuencialización de acciones y el ordenamiento de frases. 


\section{METODOLOGÍA}

Para lograr el objetivo de la investigación se realizó un diseño cuasi-experimental ABA de casos y controles con evaluación inicial (pre-test) y evaluación final (post-test). La muestra estuvo constituida por un grupo de 22 estudiantes de seis años escolarizados, que no presentaran alteraciones en su desarrollo cognitivo o motor, en un muestreo intencional no probabilístico. Los niños fueron distribuidos en 11 casos y 11 controles. En cuanto al género, ambos grupos tuvieron una participación similar de niños y de niñas $(45.5 \%$ masculino y $55.5 \%$ femenino).

\section{INSTRUMENTOS}

Para evaluar la capacidad de SP de los sujetos se optó por usar una situación problema en lugar de un test estandarizado. Este tipo de ejercicios han sido utilizados en el estudio de la SP tal como se aprecia en las investigaciones de Newell y Simon (Cit. en Carretero, García y García, 1995).

La situación escogida es una adaptación de un juego libre de Internet conocido como "Extremos", el cual consiste en llevar una pelotita desde una posición inicial hasta una posición final, moviéndola siempre de un extremo a otro ${ }^{1}$. El laberinto en la primera evaluación se puede apreciar en la Figura 1, el cuadro azul muestra la posición final de la pelota:

Figura 1. Juego "Extremos"

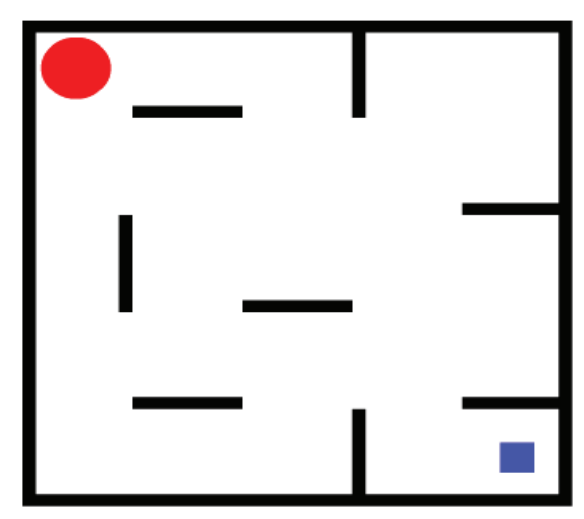

Para la evaluación post-test se usó la misma prueba con algunas modificaciones, esto con el objetivo de que la exposición al ejercicio en la primera etapa del estudio no afecte los datos. El recorrido se modifica como se observa en la Figura 2, cuidando que se pueda resolver en la misma cantidad de movimientos que el anterior (el círculo rojo indica nuevamente el punto de salida y el cuadro azul el punto de llegada).

El sitio web de donde se extrajo el juego es http://es.yupis.org/juegos/extremos/ 
Figura 2. Juego "Extremos" modificado

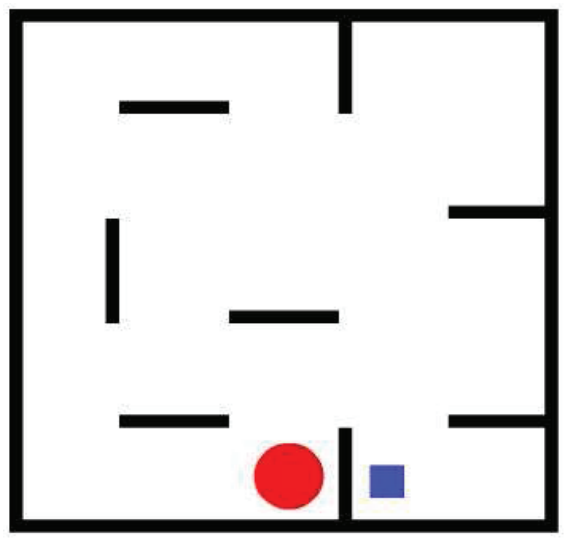

Para evitar que la exposición anterior a juegos de computador pudiese afectar el desempeño de los infantes en la prueba, se procedió a elaborar una copia a escala del laberinto, siguiendo los mismos principios que el juego original. Se optó además por usar dibujos en las paredes del laberinto para indicar los movimientos válidos, siempre que éstos coincidan se tomará como válida la acción.

Para desarrollar la situación problema, el evaluador deberá colocar la pelotita en la posición inicial y mostrarle al niño hasta dónde debe llevarla, de igual forma le explicará la forma de moverse dentro del laberinto, para lo cual se sugiere el siguiente guión: "Debes llevar la pelotita desde aquí (señalando la posición inicial) hasta acá (señala la posición final) moviéndote siempre de pared a pared, de tal forma que la pelotita siempre quede sobre uno de los dibujos (se señalan los sticker). No puedes moverte en diagonal o dando círculos, para guiarte observa atentamente los dibujos, frente a cada uno encontrarás un gemelo".

Se le muestra al niño un movimiento de ejemplo válido y un movimiento inválido para que se familiarice con la actividad. El movimiento de ejemplo debe realizarse desde una posición distinta a la inicial, esto a fin de no afectar los resultados de la ejecución de la tarea por parte del niño. Para la calificación de la prueba se tendrán en cuenta los movimientos válidos, movimientos erróneos o inválidos, el tiempo de ejecución y si la tarea fue cumplida o no.

\section{PROCEDIMIENTO}

A fin de que el contacto con la población a lo largo del desarrollo del estudio no afectara la fiabilidad de los datos recolectados, las pruebas se realizaron por un grupo de evaluadores ciegos que fueron entrenados para la aplicación de los instrumentos. A cada sujeto le fue asignado un código con el fin de mantener en secreto su identidad y prevenir la influencia de variables extrañas en el proceso de selección de los sujetos.

Luego de realizada la evaluación inicial, se asignaron al azar los miembros del grupo de control y el de casos, teniendo en cuenta como criterio de emparejamiento el género, a fin de que queden el mismo número de niños y niñas en ambos grupos. 
Una vez establecidos los grupos, los casos fueron expuestos a 10 sesiones en donde se estimularon aspectos referentes a la ubicación temporal, espacial y el uso de operadores lógicos, mediante ejercicios lingüísticos. Se tuvieron en cuenta también los aspectos prosódicos del lenguaje, por lo que en cada sesión se desarrollaron actividades como rondas o canciones. Estas sesiones tuvieron una duración aproximada de 30 a 45 minutos, y se realizaron dos veces por semana. En la Figura 3, se muestra el ejemplo de una de las sesiones de estimulación.

Figura 3. Ejemplo de sesión de estimulación

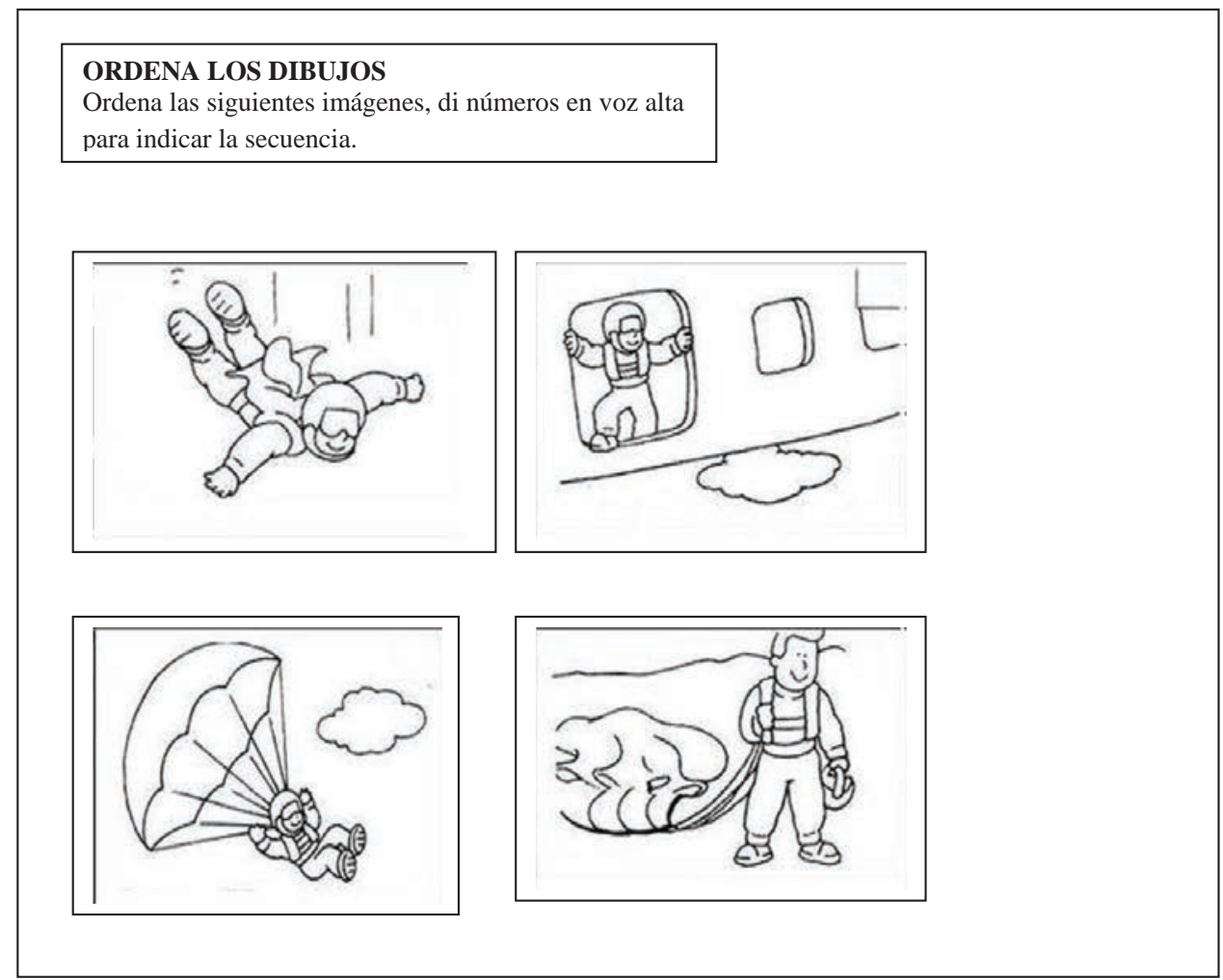

Al finalizar el programa se aplicó una prueba similar a la utilizada al principio del estudio, y se compararon los datos entre ambas mediciones; los cambios realizados en la prueba correspondieron al recorrido y a los estímulos presentados, de manera que se conserve el número de movimientos válidos mínimos para cumplir con el ejercicio.

La investigación se ciñe a los principios de la ley 1090 del psicólogo en Colombia, respetando los derechos de privacidad de los participantes y sus datos personales, los cuales no serán revelados bajo ninguna circunstancia. Las directivas del colegio permitieron la realización del estudio una vez que conocieron los aspectos referentes a éste, y los padres firmaron el consentimiento informado que autoriza la evaluación de los niños. 
EFECTIVIDAD DE UN PROGRAMA DE ESTIMULACIÓN DEL LENGUAJE SOBRE LA CAPACIDAD DE SOLUCIÓN DE PROBLEMAS DE NIÑOS DE SEIS AÑOS ESCOLARIZADOS

\section{ANÁLISIS DE LOS DATOS}

Para el análisis de los datos se utilizó el SPSS versión 18.0 teniendo en cuenta variables como: Movimientos Válidos, Movimientos Erróneos, Tiempo de Ejecución y Cumplimiento de la Tarea. Se usaron pruebas de normalidad y estadísticos de contraste para establecer diferencias en la evaluación inicial y final para cada una de las variables mencionadas, tanto para los casos como para los controles.

\section{RESULTADOS}

\subsection{CUMPLIMIENTO DE LA TAREA}

Respecto a la variable cumplimiento de la tarea, se observó que ambos grupos mejoraron respecto a la evaluación inicial, teniendo el grupo casos un desempeño del 100\% de logro en la evaluación final (Ver Tabla 1).

Tabla 1. Cumplimiento de la tarea pre-test y post-test

\begin{tabular}{|l|c|c|c|c|c|}
\hline Cumplimiento de & Casos & Controles & Cumplimiento de & Casos & Controles \\
\cline { 2 - 3 } la tarea Pre-test & $\mathrm{SI}=54.5 \%$ & $\mathrm{SI}=45.5 \%$ & larea Post-test & $\mathrm{SI}=100 \%$ & $\mathrm{SI}=81.8 \%$ \\
\cline { 2 - 3 } & $\mathrm{NO}=45.5 \%$ & $\mathrm{NO}=54.5 \%$ & & $\mathrm{NO}=0 \%$ & $\mathrm{NO}=9.1 \%$ \\
\hline
\end{tabular}

Fuente: elaboración propia.

\subsection{PRUEBA DE NORMALIDAD}

Para saber cuáles eran los estadísticos adecuados para establecer las diferencias entre la evaluación inicial y final, se utilizó la prueba de Kolmogorov-Smirnov, encontrando que los datos tienen una distribución no paramétricas (ver Tabla 2).

Tabla 2. Prueba de normalidad

\begin{tabular}{|l|c|c|c|c|c|c|}
\hline & $\begin{array}{c}\text { Movimientos } \\
\text { válidos pre }\end{array}$ & $\begin{array}{c}\text { Movimientos } \\
\text { válidos post }\end{array}$ & $\begin{array}{c}\text { Movimientos } \\
\text { erróneos pre }\end{array}$ & $\begin{array}{c}\text { Movimientos } \\
\text { erróneos post }\end{array}$ & $\begin{array}{c}\text { Tiempo } \\
\text { pre }\end{array}$ & $\begin{array}{c}\text { Tiempo } \\
\text { post }\end{array}$ \\
\hline $\mathrm{N}$ & 22 & 22 & 22 & 22 & 22 & 22 \\
\hline Media & 58,36 & 41,14 & 11,45 & 3,27 & 7,2164 & 4,2932 \\
\hline $\begin{array}{l}\text { Z de Kolmogorov- } \\
\text { Smirnov }\end{array}$ &, 942 &, 994 &, 820 & 1,208 &, 905 &, 524 \\
\hline $\begin{array}{l}\text { Sig. asintót. } \\
\text { (bilateral) }\end{array}$ &, 337 &, 276 &, 512 &, 108 &, 386 &, 946 \\
\hline
\end{tabular}

Fuente: elaboración propia. 


\subsection{CAMBIOS INTRA-GRUPO EN LAS DEMÁS VARIABLES DEL ESTUDIO}

Para el análisis intragrupos se utilizó entonces la prueba Wilcoxon (no paramétricas), encontrándose, en primer lugar, que hay diferencias significativas en el número de movimientos erróneos tanto de los niños casos como de los niños controles (en ambos grupos los movimientos erróneos disminuyeron significativamente). En segundo lugar, se encontró que sólo el grupo casos obtuvo un cambio significativo respecto al tiempo usado para resolver la tarea, lo que quiere decir que después de la estimulación los niños lo hicieron en menos tiempo del que ellos mismos usaron en la primera evaluación, mostrando una mejoría al interior del grupo, pues en los controles no hay cambio significativo (ver Tabla 3).

Tabla 3. Cambios intra-grupo

\begin{tabular}{|l|l|c|c|c|c|c|c|}
\hline \multirow{2}{*}{ Procesos cognitivos } & \multirow{2}{*}{ Grupos } & \multicolumn{2}{|c|}{ Pre-test } & \multicolumn{2}{c|}{ Post-test } & \multicolumn{2}{c|}{ Wilcoxon } \\
\cline { 3 - 8 } & & Media & DT & Media & DT & Z & Sig (p) \\
\hline \multirow{2}{*}{ Movimientos Válidos } & Casos & 64,27 & 57,250 & 38,91 & 25,217 & $-1,172$ &, 241 \\
\cline { 2 - 8 } & Controles & 52,45 & 42,302 & 43,36 & 52,391 &,- 667 &, 505 \\
\hline \multirow{2}{*}{ Movimientos Erróneos } & Casos & 10,36 & 9,882 & 2,64 & 1,859 & $-2,313$ &, 021 \\
\cline { 2 - 8 } & Controles & 12,55 & 11,379 & 3,91 & 4,763 & $-2,268$ &, 023 \\
\hline \multirow{2}{*}{ Tiempo } & Casos & 7,0255 & 3,90258 & 4,0718 & 2,12711 & $-2,312$ &, 021 \\
\cline { 2 - 8 } & Controles & 7,4073 & 4,70855 & 4,5145 & 3,25013 & $-1,423$ &, 155 \\
\hline
\end{tabular}

Fuente: elaboración propia.

\subsection{CAMBIOS INTER-GRUPO}

Para el análisis de los cambios del grupo casos respecto al grupo controles se usó la U de Mann Whitney, encontrándose que no hay diferencias significativas del grupo casos respecto al grupo controles, aunque en el apartado anterior el grupo casos sí tuvo una diferencia significativa respecto a sí mismo en el tiempo para resolver la tarea (ver Tabla 4).

Tabla 4. Cambios inter-grupo

\begin{tabular}{|c|c|c|c|c|c|c|c|}
\hline \multirow{2}{*}{ Procesos cognitivos } & \multirow{2}{*}{ Grupos } & \multicolumn{2}{|c|}{ Pre-test } & \multicolumn{2}{|c|}{ Post-test } & \multicolumn{2}{|c|}{ U De Mann Whitney } \\
\hline & & Media & DT & Media & DT & $\mathbf{Z}$ & Sig (p) \\
\hline \multirow{2}{*}{ Movimientos Válidos } & Casos & 64,27 & 57,250 & 38,91 & 25,217 & \multirow{2}{*}{,- 855} & \multirow{2}{*}{, 393} \\
\hline & Controles & 52,45 & 42,302 & 43,36 & 52,391 & & \\
\hline \multirow{2}{*}{ Movimientos Erróneos } & Casos & 10,36 & 9,882 & 2,64 & 1,859 & \multirow{2}{*}{,- 033} & \multirow{2}{*}{1,000} \\
\hline & Controles & 12,55 & 11,379 & 3,91 & 4,763 & & \\
\hline \multirow{2}{*}{ Tiempo } & Casos & 7,0255 & 3,90258 & 4,0718 & 2,12711 & \multirow{2}{*}{,- 164} & \multirow{2}{*}{,870 } \\
\hline & Controles & 7,4073 & 4,70855 & 4,5145 & 3,25013 & & \\
\hline
\end{tabular}

Fuente: elaboración propia. 
Estudios Pedagógicos XL, $\mathrm{N}^{\circ}$ 2: 361-372, 2014

EFECTIVIDAD DE UN PROGRAMA DE ESTIMULACIÓN DEL LENGUAJE SOBRE LA CAPACIDAD DE SOLUCIÓN DE PROBLEMAS DE NIÑOS DE SEIS AÑOS ESCOLARIZADOS

\section{DISCUSIÓN Y CONCLUSIONES}

Dentro de los resultados más llamativos de la presente investigación se encuentra que cuando se hacen las comparaciones intragrupales, tanto los niños casos como los niños controles cometieron significativamente menos movimientos erróneos, pero que los niños casos a diferencia de los controles, lograron ejecutar la tarea en menos tiempo. Este cambio observado puede obedecer a varias razones.

Primero, pudieron producirse cambios en el espacio del problema, es decir, en la representación del ambiente de la tarea, dichos cambios pueden ser tanto de tamaño como de complejidad, cabe recordar el argumento de Simon según el cual la facilidad con la que se resuelva un problema dependerá en gran medida del éxito de su representación.

Segundo, una mejora en el uso de los operadores al interior del espacio del problema les pudo permitir a los niños del grupo de casos aumentar su velocidad en la resolución, es decir, el espacio del problema en ambos grupos pudo tener características similares difiriendo sólo en la velocidad de pasar de un nodo a otro, de un estado de conocimiento a otro; esto supone también un mejor uso de la memoria a corto plazo, o posiblemente de la memoria de trabajo.

Dado que ninguno de los panoramas planteados es excluyente entre sí, no se puede descartar una reducción del espacio del problema acompañado de una mejora en el uso de los operadores. Los cambios observados en los grupos parecen obedecer a componentes instrumentales de la SP, mas no sugieren un cambio en el tipo de razonamiento utilizado por los niños; esto se puede decir gracias a que las variables de Movimientos Válidos y Movimientos Erróneos no tuvieron cambios atribuibles al programa.

De acuerdo con lo anterior y en línea con las teorías de la psicología histórico-cultural, el lenguaje aparece como un elemento externo cuya interiorización permite la aparición y la evolución de los procesos cognitivos superiores. La capacidad de organizar planes de acción antes de ejecutarlos directamente, le otorga al sujeto la oportunidad de escapar de la experiencia inmediata y ordenar su comportamiento a través del tiempo, haciéndolo más eficiente y mostrando una vez más las relaciones entre pensamiento y lenguaje.

Para Vigotsky (1995), el pensamiento no sólo se expresa a través del lenguaje, sino que encuentra su forma en éste. Según este autor, estos conceptos forman una amalgama tal, que sería muy complicado concebir el pensamiento sin el lenguaje. La estructura gramatical moldea la forma en la que se representa el mundo en la mente del sujeto, de modo que utiliza las formas y estructuras gramaticales antes de comprender la lógica que las sustenta, es decir, "domina antes la sintaxis del lenguaje que la del pensamiento" (1995: 74).

En ese orden de ideas, Montealegre (2007) afirma que el lenguaje es como una herramienta tal como los ojos y las manos en la solución de una situación problemática, debido a que prepara instrumentos que puedan ser útiles en la solución de la tarea, y permite planear acciones futuras de modo que puede ir más allá de las experiencias anteriores, además de regular el comportamiento haciendo más eficiente la puesta en acción del plan construido.

Así, el lenguaje es un instrumento que el sujeto utiliza en la solución del problema y no sólo en la construcción de su representación; cuando un sujeto crea un espacio del problema basado en la interpretación lingüística de las instrucciones y las tareas a realizar, se basa en los operadores disponibles que dicha interpretación le proporciona para poder moverse por los nodos de éste y encontrar así la solución. Dichos operadores pueden ser movimientos, palabras, operaciones matemáticas, construcción de herramientas; los operadores y su uso dependerán del tipo de problema planteado y de los movimientos lícitos. 
Los ejercicios utilizados en las sesiones de estimulación estuvieron en su mayoría enfocados en ordenar palabras o secuencias de acciones, teniendo en cuenta que la secuencialización es parte importante en la resolución de problemas. Al parecer, el uso del lenguaje en este tipo de actividades y la práctica prolongada de estos ejercicios se encuentra relacionada con una mejora en el tiempo usado en la solución de problemas, bien sea por un mejor uso de los operadores lógicos al interior del espacio del problema, o por modificaciones del tamaño y complejidad del mismo.

Sin embargo, en la investigación no aparecen cambios significativos en el análisis intergrupal, ello puede deberse al tiempo corto de exposición y a usar un programa de estimulación del lenguaje por fuera del currículo y no insertado en él. Las afirmaciones anteriores se derivan del conocimiento en programas de estimulación de habilidades de pensamiento en tres metodologías específicas.

- El tipo Bolt-on interviene las habilidades de pensamiento separadas de otras habilidades y del currículo, como ocurre con el programa Instrumental Enrichment (IE) desarrollado por Feuerstein, Hoffman \& Miller (1980), Savell, Twohig \& Rachford (1986) y Blagg (1991).

- La metodología Embedded centra la estimulación del pensamiento en temas específicos del currículo, por ejemplo el Cognitive Aceleration Through Science Education (CASE) desarrollado por Adey (1992), Adey \& Shayer (1993) y Shayer \& Adey (1993).

- Por último, se encuentran los que promueven habilidades de pensamiento por medio de acciones que son totalmente insertadas en el currículo denominados Infusion, tal como el programa Activating Children's Thinking Skills (ACTS) desarrollado por Swartz \& Parks (1994) y McGuinness (1993).

Los tres tipos de programas han mostrado efectividad, pues las habilidades de pensamiento resultan beneficiadas después de la ejecución de los mismos. Sin embargo, los programas de tipo Embedded e Infusion muestran mayores ventajas respecto a los Bolt-on, porque las habilidades de pensamiento se tornan transversales a toda la vida académica, afianzado de esa forma la toma de decisiones, la resolución de problemas, la búsqueda de sentido a las actividades escolares y, en general, el razonamiento como vía de comprensión del mundo y de las relaciones. Además, los investigadores hablan de tiempos de exposición de mínimo dos años para alcanzar cambios mucho más estables y significativos. Esta investigación sólo tuvo 5 semanas de estimulación con los niños, por ello lo más recomendable es replicar estudios como éstos con un tiempo de exposición mucho más prolongado, que permita evaluar con mayor claridad la influencia de este tipo de programas sobre las variables estudiadas.

\section{REFERENCIAS BIBLIOGRÁFICAS}

Adey, P. \& Shayer, M. (1993). An Exploration of Long Term Far-transfer Effects Following an Extended Intervention Program in the High School Curriculum. Cognition and Instruction, vol. 11, n. 1, 1-29.

(1992). The CASE Results: Implications for Science Teaching. International Journal for Science Education, vol. 14, n. 2, 137-146.

Ardila, A., Ostrosky-Solís, F. (2008). Desarrollo histórico de las funciones ejecutivas. Revista Neuropsicología, Neuropsiquiatría y Neurociencias, vol. 8, n. 1, 1-21. 
Blagg, N. (1991). Can We Teach Intelligence? Hilisdale: Erbaum.

Carretero, M. y Asensio, M. (Coords.). (2004). Psicología del pensamiento. Madrid: Alianza Editorial.

Dewey, J. \& Bento, J. (2009). Activating Children's Thinking Skills (ACTS): The Effects of an Infusion Approach to Teaching Thinking in Primary Schools. British Journal of Educational Psychology, vol. 79, n. 2, 329-351.

, García, M. y García, J. (1995). Lecturas de psicología del pensamiento. Razonamiento, solución de problemas y desarrollo cognitivo ( $2^{\mathrm{a}}$ Ed.). Madrid: Alianza Editorial.

Fahim, M. \& Pezeshki, M. (2012). Manipulating Critical Thinking Skills in Test Taking. International Journal of Education, vol. 4, n. 1. Recuperado de http://www.macrothink.org/journal/ index.php/ije/article/view/1169, consultado en septiembre de 2012.

Feuerstein. R., Hoffman. M. B. \& Miller, R. (1980). Instrumental Enrichment: An Intervention for Cognitive Modifiability. Baltimore: University Park Press.

Kintsch, G. \& Greeno, J. (1985). Understanding and Solving Word Arithmetic Problems. Psychological Review, vol. 92, n. 1, 109-129.

McGuinness, C. (1993). Teaching Thinking: New Signs for Theories of Cognition. Educational Psychology, vol. 13, n. 3, 305-316.

Molina, D. (2009). La génesis de los procesos psíquicos, una aproximación desde el modelo neuropsicológico de Luria. Poiésis. Revista Electrónica de Psicología Social, n. 18, 1-5.

Montealegre, R. (2007). La solución de problemas cognitivos. Una reflexión cognitiva sociocultural. Avances en Psicología Latinoamericana, vol. 25, n. 2, 20-39.

Piaget, J. (1991). Seis estudios de psicología. Barcelona: Editorial Labor.

Rains, D. (2003). Principios de neuropsicología humana. México D.F.: McGraw-Hill.

Rich, J., Miller, D. \& DeTora, L. (2011). From Concept to Application: Student Narratives of Problem-solving as a Basis for Writing Assignments in Science Classes. Across the Disciplines, vol. 8, n. 1. Recuperado de http://wac.colostate.edu/atd/articles/richetal2011.cfm, consultado en septiembre de 2012 .

Rohde, T. \& Thompson, L. (2007). Predicting Academic Achievement with Cognitive Ability. Intelligence, vol. 35, n. 1, 83-92.

Savell, J. M., Twohig, P. T. \& Rachford, D. L. (1986). Empirical Status of Feuerstein's "Instrumental Enrichment" (FIE) Technique as a Method of Teaching Thinking Skills. Review of Educational Research, vol. 56, n. 4, 381-409.

Setti, A. \& Caramelli, N. (2007). Inhibition and Language Pragmatic View in Redundant Data Problem Solving. Cognitive Development, vol. 22, n. 3, 299-309.

Shayer, M. \& Adey, P. (1993). Accelerating the Development of Formal Thinking in Middle and High School Students IV: Three Years after a Two-year Intervention. Journal of Research in Science Teaching, vol. 30, n. 4, 351-366.

Swartz, R. \& Parks, S. (1994). Infusing the Teaching of Critical and Creative Thinking into Content Instruction: A Lesson Design Handbook for the Elementary Grades. California: Critical Thinking Press and Software.

Vygotsky, L. (1995). Pensamiento y lenguaje. Buenos Aires: Paidós. 